\title{
A protocol paper: community engagement interventions for cardiovascular disease prevention in socially disadvantaged populations in the UK: an implementation research study
}

Papreen Nahar ${ }^{1 *}$ (D) Harm van Marwijk², Linda Gibson ${ }^{3}$, Geofrey Musinguzi ${ }^{4,5}$, Sibyl Anthierens ${ }^{5}$, Elizabeth Ford², Stephen A. Bremner ${ }^{2}$, Mark Bowyer ${ }^{3}$, Jean Yves Le Reste ${ }^{6}$, Tholene Sodi ${ }^{7}$ and Hilde Bastiaens ${ }^{5}$

\begin{abstract}
Background: Cardiovascular disorders (CVD) are the single greatest cause of mortality worldwide. In the UK, the National Health Service (NHS) has launched an initiative of health checks over and above current care to tackle CVD. However, the uptake of Health Checks is poor in disadvantaged communities. This protocol paper sets out a UK-based study (Sussex and Nottingham) aiming to co-produce a community delivered CVD risk assessment and coaching intervention to support community members to reduce their risk of CVD.

The overall aim of the project is to implement a tailored-to-context community engagement (CE) intervention on awareness of CVD risks in vulnerable populations in high, middle and low-income countries. The specific objectives of the study are to enhance stakeholder' engagement; to implement lifestyle interventions for cardiovascular primary prevention, in disadvantaged populations and motivate uptake of NHS health checks.

Methods: This study uses both qualitative and quantitative methods in three phases of evaluation, including pre-, per- and post-implementation. To ensure contextual appropriateness the 'Scaling-up Packages of Interventions for Cardiovascular disease prevention in selected sites in Europe and Sub-Saharan Africa: An implementation research' (SPICES) project will organize a multi-component community-engagement intervention. For the qualitative component, the pre-implementation phase will involve a contextual assessment and stakeholder mapping, exploring potentials for CVD risk profiling strategies and led by trained Community Health Volunteers (CHV) to identify accessibility and acceptability. The per-implementation phase will involve healthy lifestyle counselling provided by CHVs and evaluation of the outcome to identify fidelity and scalability. The post-implementation phase will involve developing sustainable community-based strategies for CVD risk reduction. All three components will include a process evaluation. A stepped wedge cluster randomised trial of the roll out will focus on implementation outcomes including uptake and engagement and changes in risk profiles. The quantitative component includes pre and post-intervention surveys. The theory of the socio-ecological framework will be applied to analyse the community engagement approach.

Discussion: Based on the results ultimately a sustainable community engagement-based strategy for the primary prevention of CVD risk will be developed to enhance the performance of NHS health care in the UK. The Trial Registration number is ISRCTN68334579.
\end{abstract}

Keywords: Implementation research, CVD risk prevention, Community engagement, Stepped-wedge cluster randomised trial

\footnotetext{
* Correspondence: P.Nahar@bsms.ac.uk

'Department of Global Health and Infection, Brighton and Sussex Medical

School, The University of Sussex, Brighton, UK

Full list of author information is available at the end of the article
}

(c) The Author(s). 2020 Open Access This article is distributed under the terms of the Creative Commons Attribution 4.0 International License (http://creativecommons.org/licenses/by/4.0/), which permits unrestricted use, distribution, and reproduction in any medium, provided you give appropriate credit to the original author(s) and the source, provide a link to the Creative Commons license, and indicate if changes were made. The Creative Commons Public Domain Dedication waiver (http://creativecommons.org/publicdomain/zero/1.0/) applies to the data made available in this article, unless otherwise stated. 


\section{Introduction}

Cardiovascular disorders (CVD) are the single greatest cause of mortality worldwide each year, estimated to contribute to $31 \%$ of all deaths globally [1]. Tackling CVD is an international priority and there have been many global initiatives such as the "Global Hearts" programme, a package launched by the World Health Organisation (WHO) and partners, to enhance the prevention and control of CVD. Some risk factors for CVD are non-modifiable, such as age, ethnicity and family history [2]. Some other risk factors for CVD are modifiable, such as smoking, a lack of physical activity, being overweight, lower consumption of fruit and vegetables, high blood pressure, diabetes and high cholesterol [2]. These risk factors can be changed through lifestyle or behavioural modifications. There is evidence of a social gradient in the prevalence of CVD, which points to associations between social and financial deprivation, vulnerability and risk factors for CVD [3]. In 2015, CVD was the leading cause of mortality in the context of all chronic diseases, accounting for 27 and $25 \%$ of deaths in men and women respectively, in the UK [2]. Coronary heart disease (CHD) and stroke were the main CVDs responsible for this mortality of men and women across all ages. As per British Heart Foundation report in 2017 CVD has a huge financial burden with annual associated healthcare costs estimated to be $£ 9$ billion annually in the UK [2]. The UK has a standardised CVD death rate of 265.1 per 100,000 [2].

In the UK, the National Health Service (NHS) has launched the Health Check initiative aimed to reduce CVD. It is a national risk assessment and management programme, free to adults aged 40 to 74 living in England, who do not currently have any vascular disorders and are not being treated for certain risk factors such as diabetes [4]. It aims to assess the 10-year risk of $\mathrm{CV}$ events and disorders. Risk is assessed using QRISK2 [5], a tool which involves collection of the following information: age, gender, ethnicity, smoking status, family history of CHD, body mass index (BMI), cholesterol test, systolic and diastolic blood pressure, levels of physical activity, and alcohol consumption. Attendees receive a low ( $<10 \%$ chance of event in 10 years), medium $(>10 \%$ but $<20 \%$ ), or high (>20\%) 10-year cardiovascular (QRISK2) score. Above the 10\% cut-off, attendees are offered a discussion with a qualified person, such as a nurse, about lifestyle and motivation to change, which may include goal setting and plans for follow up. Patients may also be offered medication for cholesterol and blood pressure. The NHS Health Check is recommended to be undertaken every 5 years.

Modelling predicted that the NHS Health Check could prevent 1600 heart attacks and strokes each year if implemented as intended [6]. Whilst evidence suggests that the Health Check programme has the potential to reduce CVD events and has therefore been rolled out nationally across the UK, its implementation has been poor, especially in some of the most disadvantaged groups at highest risk of developing CVD. In 2014, Public Health England (PHE) issued a call for action to increase the uptake rate of NHS Health Checks to 75\% [7] and to increase awareness of risk and engagement with existing resources. Yet, as of 2017, current uptake remains far from this target with current predictions suggesting only $40 \%$ of the eligible population will receive one [8], due to the fact that uptake is low (48\%) even when Health Checks are offered $[8,9]$.

Data from some regions with very large ethnic minority communities and socio-economically challenged populations showed that only $45 \%$ of patients who were invited for the check attended and subsequently received some form of counselling when they needed it. The current study uses the term socio economically challenged (SEC) or disadvantaged population based on a study where SEC was assessed using the Townsend score using 2001 census-derived measures of overcrowding, car ownership and education available at lower super output area. This has been found by linking the individuals' postcode to lower super output area, approximately 150 households [10]. Authors have also suggested that higher uptake in SEC communities would reduce the possibility of exacerbation of inequalities [10]. Difficulty with accessing general practices, especially among socially vulnerable groups, has been highlighted as a common barrier to attendance at Health Checks [11]. A community-based engagement approach, which takes the CVD risking profiling and affiliated advice processes outside of the formal healthcare facility setting, has the potential to improve access to Health Checks and could be an effective and scalable way for improving the implementation and uptake of Health Checks. Community engagement $(\mathrm{CE})$ has been conceptualised as "the process of working collaboratively with and through groups of people affiliated by geographic proximity, special interest, or similar situations, to address issues affecting the well-being of those people" [12]. A review of community engagement interventions found them to be effective in improving health behaviours (such as physical activity), health consequences and psychological outcomes (i.e. selfefficacy and perceived social support) [13]. Communitybased intervention programmes have been implemented to increase the uptake of cancer screening programmes. The programmes have been found to be effective in increasing outcomes such as recognition, receipt and maintenance of screening behaviours [14]. The CE approach offers the opportunity for task-shifting and owning the programme, whereby trained non-healthcareprofessionals can perform CVD risk profiling assessments to individuals who might not otherwise be captured by the formal care pathway. 
There is evidence that CVD risk assessments can be successfully delivered by Community Health Workers (CHWs), outside or inside the healthcare system. An observational study conducted in Bangladesh, Guatemala, Mexico and South Africa has demonstrated that CHWs who are inhabitants of their local communities and were fluent in the community's predominant language, can perform community-based screenings to predict CVD risk as effectively as physicians and nurses when using the non-laboratory-based Gaziano CVD risk scoring tool [15]. CHWs were trained for 1-2 weeks, and results showed a 96.8\% agreement between risk scores assigned by CHWs and healthcare professionals. However, a question remains whether the model taken in the global South could be transferrable to the global North, but it is at least plausible that a community-based engagement approach will be effective for increasing the uptake of CVD risk assessment, particularly in disadvantaged communities of the global North. There are examples in the global North on community engagement in health [16], and indeed the voluntary or 'third sector' have been considered key partners in the delivery of health promotion initiatives in the community [17].

Authors have argued that because of the current economic constraints with the formal healthcare system in the UK, the focus should be upon supplementing a service delivery model with an alternative community development model [18]. The key aspect is supplementing formal service delivery by utilizing communities' 'social capital'. The term 'social capital' describes the various resources that people may have through their relationships in families, communities and other social networks. Social capital bonds people together and helps them make links beyond their immediate friends and neighbours [19].

For this compassionate community approach to work, contextual appropriateness and cultural sensitivity of an intervention is crucial [20]. Following this argument, the SPICES project (Scaling-up Packages of Interventions for Cardiovascular disease prevention in selected sites in Europe and Sub-Saharan Africa) in two areas of England, East Sussex and Nottingham, will co-produce a multi-component communityengagement intervention focussed on delivering a Health Check-style CVD risk screening intervention. The UK SPICES project will use appropriate health coaching and follow-up, in a community setting [21] delivered by community volunteers. The specific objectives of the project are:

1. To explore with stakeholders the potential for a community engagement-based CVD primary prevention programme to support or enhance the NHS Health Check Programme.
2. To co-produce with the communities an evidenceinformed community-engagement intervention on CVD risk, based on the NHS Health Check model, tailored to the context in disadvantaged communities in East Sussex and Nottingham.

3. To implement the intervention in the local communities where it was co-produced, and:

- assess its effectiveness versus routine care.

- assess the fidelity, feasibility, acceptability, uptake and scalability of the implementation.

- carry out a process evaluation of the intervention and its implementation.

The SPICES Project is a Horizon 2020 project financed by the European Commission that aims to address the CVD burden [22]. The overall objective is to implement and evaluate a comprehensive cardiovascular disease (CVD) prevention and care program at the community level in five countries (Belgium, France, South Africa, Uganda, and UK), to identify and compare barriers and facilitators for implementation across study contexts and to develop a learning community. The intervention will be trialled and evaluated using a mixed methods approach using both qualitative and quantitative methods.

\section{Methods}

\section{Theoretical model}

SPICES is underpinned by the Consolidated Framework for Advancing Implementation Research (CFAIR; 23), and Reach, Effectiveness, Adoption, Implementation, and Maintenance (sustainability) framework /RE-AIM models [23]. It is a global health project, and thus needs a socio-ecological framework for consistency between teams [24]. This framework allows an understanding of the multifaceted and interactive effects of personal, social and environmental factors that determine behaviour; and for identifying behavioural and organisational leverage points and intermediaries for health promotion within organisations and communities.

\section{Study design}

A mixed-methods research methodology will be applied, strategically combining qualitative and quantitative methods at both sites. This approach will allow us to model the iterative nature of coproduction and implementation research without compromising the rigour of the study $[25,26]$. The study will take place in three phases:

- Pre-intervention; when stakeholder mapping and local adaptation will be carried out.

- Per-intervention roll out, recruitment and evaluation. 
- Post-intervention evaluations and feedback [27] Process evaluation will be conducted in all three phases.

\section{Processes}

Stage 1: to explore the implementation context and coproduce the intervention

To explore the context where the implementation will take place we will carry out several mappings. These will give us the context for recruitment and implementation co-design.

They are as follows:

(a) Mapping the potential stakeholders: Mapping of the stakeholders will be done to find out who are the key stakeholders, where they come from, and what they are looking for in relationship to the study objectives [28]. To engage the community, it is essential to map the community stakeholders (civil society organisations) as they are the gatekeepers of the community. Three levels of stakeholder mapping will be carried out, namely at macro, meso and micro levels.

Macro-level: Stakeholders will be identified via the existing link of PI of the project in the community through meetings with local public health or other relevant departments and CSOs and using online information. Interviews with this category of stakeholders will provide insights into implementation sustainability.

Meso-level: A strategic community volunteer organisation mapping will be carried out to find out the relevant organisations, through which individual volunteers will be selected. This will be done in three ways; using online searches, personal contacts and snowballing. In-depth interviews will be conducted to co-design a sustainable intervention implementation. Micro-level: An exploration will be undertaken with volunteers and end-user groups to co-design an acceptable and feasible intervention implementation.

(b) Mapping the context: social mapping will be carried out to explore the lifestyle context of the community via observations.

(c) Training of volunteers by professional health trainers and researchers following the current NICE Public health guideline [PH6] 'Behaviour change: general approaches' [29].

(d) CVD risk profiling by trained community health volunteers $(\mathrm{CHV})$ during the pre-implementation stage.

CHVs will be the persons who have been involved in health-related volunteering for example volunteers who worked in cancer prevention, health check, healthy lifestyle etc. programme. They will be involved in the screening of the CVD risk population and implement the designed intervention.

\section{Expected intervention}

The final elements of the intervention will be coproduced within each community setting, following the mapping exercises outlined above on the contextual barriers and enablers of intervention implementation. Data from each site will contribute to a holistic intervention. These four sites are geographically close to each other and have similarities in socio-economic status so it is likely that the interventions will be similar. However, although the core components are similar, implementation within a setting/organization could be different. As outlined in the CFAIR [30], interventions are usually composed of a core component which is essential and indispensable, and an adaptable periphery, which can and should be tailored to the specific setting and users.

Core components Following identification of moderate to high risk for CVD, the intervention will consist of non-clinical (non-NHS) individual or group support sessions within the community, focus on motivating behaviour change. Each participant will be supported by trained SPICES researchers or community health workers to identify behaviour change goals, produce action plans to achieve them, and problem solve in cases of unexpected outcomes. All SPICES Interventions are theoretically grounded in the theory of behaviour change and deploy the strongest evidenced Behaviour Change Techniques (BCTs) from the literature: Goal Setting, Action Planning, Problem Solving, Motivational Interviewing, Feedback on progress towards goals, Feedback on the health impact. The use of these six BCTs are focussed in SPICES on five Target Behaviours: Reduce/cease smoking, Increase moderate physical activity, Reduce the fat, salt, and sugar content of the diet, Increase fibre, oily fish, fruit and vegetable content of the diet, Reduce sedentary hours.

Community adaptation The exact elements of the support sessions will be tailored to individuals and their community context, will be determined during iterative co-design with community representatives, and will be drawn from the following [31, 32]:

Step-I - Goal setting

Every participant should receive specific healthy lifestyle counselling/feedback based on their individual item InterHEART assessment scores (the moderate group). The feedback will be based on a review of international guidelines 
conducted as formative work for the SPICES project intervention [33]. SPICES behaviour change support sessions will be based on the best-evidenced approaches to healthy lifestyle modification and community context and preferences.

The following screening self-report questionnaires assess the benefit of possible behaviour changes in relation to physical activities and dietary practices. Selected modified questionnaires will be used for measuring the impact of implementations;

1) International Physical Activity Questionnaire (http://www.sdp.univ.fvg.it/sites/default/files/IPAQ_ English_self-admin_long.pdf) is an internationally validated instrument to capture information about weekly physical activity habits, behaviours and routines.

2) The Dietary Approaches to Stop Hypertension Questionnaire DASH-Q is a self-reporting lifestyle questionnaire (https://www.bmj.com/content/357/ bmj.j1794.full.print) to capture information about weekly dietary habits, routines and behaviours, based around 'Dietary Approach to Stopping Hypertension' [34].

3) Current behaviours audit: Using food and physical activity diaries prepared by and provided to participants by the SPICES research team, participants will be encouraged to complete an audit of 1 week of current dietary and physical activity behaviours, habits and routines to establish a baseline from which goals for change and improvement can be set in negotiation with SPICES CHVs.

4) The Attitudes and Beliefs about Cardiovascular Disease (ABCD) Risk Questionnaire, licenced under Creative Commons Attribution 4.0 International Licence, developed by Maria Woringer et al. [35]. to assess participant perception of personal heart health risk.

5) The EQ-5D-5 L internationally validated Quality of Life self-reporting questionnaire.

Step-II - Action planning by the participants

Participants will be asked to create an action plan with appropriate goal setting for two behaviours (diet and exercise habits) in relation to when, where and how they will undertake, for example, physical activity (based on the item stems used by Luszczynska \& Schwarzer [36]; when the physical activity will be performed, where it will be performed, how often it will be performed. The way goals are reached and plans recorded will be co-designed with key stakeholders.

Step III - Problem-solving
CHVs will help participants to analyse any factors which may influence their ability to achieve the goals and to generate strategies which could help them overcome these barriers.

CHVs will use Motivational Interviewing techniques about health, social and environmental, and emotional barriers and consequences. Culturally and contextsensitive information will be provided (both verbally and in the form of leaflets) about the importance of eating healthily, being physically active, and not smoking for positive outcomes on physical and mental health.

\section{Stage 2: intervention roll out, recruitment and evaluation}

This will be an open-label, stepped wedge cluster randomised controlled trial, examining fidelity, feasibility, acceptability, uptake and scalability of the intervention.

\section{Eligible population and setting}

Economically disadvantaged, lower socio-economic status (SES) postcodes, will be identified using the overall Index of Multiple Deprivation [37]; Participants' SES will be determined by their postcode of residence. Any resident aged 18 or above living in the study postcode areas will be eligible to take part in the baseline assessment for the study.

\section{(a) Study sample size and power calculation}

The sample size calculation for the quantitative study used statistical modelling for a stepped wedge design, randomising community centres over time with the InterHEART score as the outcome (90\% power for $5 \%$ significance, small effect size (Cohen's D) $=0.25$, intracluster correlation coefficient of 0.05 , control clusters crossing to intervention in 4 steps, participant autocorrelation $=0.7$ and cluster autocorrelation $=0.9$ ), which requires a total of at least 144 persons. This needs approximately 200-300 people across the two sites as we expect a high level of attrition (as much as 50\%). At least 1500 community members will need to be screened to achieve this recruitment [38].

(b) Recruitment of community health volunteers and trial participants

Community Health Volunteers (CHVs) will be recruited to perform CVD risk profiling assessments through a combination of 'doorstep outreach' and 'intermediary organisation recruitment' approaches in East Sussex and through existing community and neighbourhood groups with the assistance of partners such as Self-Help UK, the Renewal Trust, Nottingham CVS and others in Nottingham. For recruitment of trial participants, we will use similar community networks, and endeavour to use quota sampling, in that we will seek to ensure the inclusion of high, low and median income neighbourhood residents, citizens from the 
South Asian and African diasporas; and will encourage participants to refer others to the researchers who may be able to potentially contribute or participate in the study.

(c) Baseline screening of CVD risk

Participants will fill in the validated non-laboratory based InterHEART score to determine suitability for the trial. The InterHEART scoring tool requires minimal resources which is practical for use within the community. There is also evidence to suggest that the InterHEART can reliably predict the incidence of CVD and death in low, middle, and high-income countries for a mean follow-up of 4.1 years [39]. Risk is expressed as a score from the InterHEART: 0-9 (Low risk), 10-15 (moderate risk), and 16-48 (high risk). The InterHEART scoring tool will be inserted onto a mHealth platform so that the trained CHVs can easily administer them during community engagement and contact, and online data will directly reach the University repository in real time from the respondents' device. The moderate risk (amber) score population will be selected for participation in the intervention ( $=$ score of 10 or higher), and will fill out the self-completion survey InterHEART scoring every 3 months [40].

\section{(d) Clinical outcome and follow-up}

The primary outcome will be the change in the CVD risk score among people who complete the community delivered CVD risk assessment and coaching (before/ after and between groups). In addition, a by-product outcome will be gathered from participants identified as 'high risk' (who are not part of the sample) during the screening process. This group of participants will be signposted to their GP surgery requesting a) a formal CVD risk check-up and b) completed NHS Health Checks. The following secondary outcomes will be gathered from such participants identified as 'high risk': 1) Numbers of participants who a) self-referred (defined as having contacted their GP surgery requesting for a formal check-up) and b) completed the NHS Health Check, 2) Self-reported lifestyle risk factors gathered through survey instruments and interviews, 3) Observed data on all participants' age, gender, ethnicity, postcode, hip to waist ratio, gathered by trained volunteers. Quantitative analysis of changes in behavioural intention, target behaviours, and measurable CVD risk. The outcome will be assessed at multiple time points after the interventions, once every 3 months, to see whether the behavioural change has been sustained. The per-implementation phase data will provide an insight of the challenges of implementation. Amendment in the design of implementation may tailored accordingly. (e) Post-intervention qualitative evaluation and feedback

In the post-intervention phase, a qualitative evaluation will be carried out during which the following implementation parameters will be assessed:

1. The impact on awareness of CVD risks and mitigating measures, amongst disadvantaged populations of a community-based, non-clinical, CVD risk scoring tool and education.

2. The impact of the community based non-clinical CVD risk scoring tool and education on motivational healthy lifestyle among disadvantaged populations.

3. The facilitators and barriers to the adoption of a community-based CVD prevention implementation programme, by target populations.

4. The perspectives of participants regarding their experience and meaning of the intervention.

These will be explored with a subset of intervention participants using focus groups or/and in-depth interview and community mapping. Participants for the qualitative component will include adult volunteers, public health stakeholders and people within the community. The community volunteers will be selected via community organisations and public health stakeholders will be selected from the same area of the research site. Community participants for the qualitative component will be selected via the community volunteers. This post-intervention qualitative study will include purposively selected trial participants.

The number of participants for the qualitative component will be flexible. The number will be determined through the principles of saturation and diversity. However, from each site, we will aim to include at least 12 respondents and a maximum of 30 respondents from different categories $[41,42]$.

\section{Stage 3: process evaluation of the intervention}

To assess the fidelity of the conclusions concerning the project's effectiveness, ongoing assessment, monitoring, and enhancement is important. If significant intervention effects are observed, but fidelity was not assessed, it cannot be determined if the effectiveness is attributable to unintentionally added or omitted components. Bellg and colleagues [43] propose that considerations of fidelity should permeate all stages of the study: design of the study, provision of training, delivery of the intervention, receipt of the intervention, and re-enactment of skills. As a result, we will carry out a process evaluation of the project. This will be reached through Process documentation of all the stages of this project including 
community volunteers mapping, healthy lifestyle counselling, action planning and problem-solving.

Thirsk and Clark [44] argue how health-care interventions need to be understood in ways that are responsive to the complexities and intricacies of programs, people and places. They emphasise the understanding of the comprehensive experience of the persons who are delivering and receiving the intervention. Process evaluation is a tool that can capture the intervention experience. We will be following the model designed by Moore et al. [45]; Fig. 1.

\section{Data analysis}

Flow of trial clusters and participants will be depicted in a CONSORT 2010 Statement flow diagram from the extension to trials randomised in clusters [46]. Quantitative data will be analysed using Stata version 16 or later [47]. Descriptive statistics will summarise outcomes before and after clusters cross over to the intervention [48]. Normally distributed variables will be summarised by means and standard deviations, skewed continuous variables by medians and interquartile ranges, categorical variables by frequencies and percentages. We will estimate the treatment effect using a cross-classified linear mixed effects model. A statistical analysis plan will be agreed and signed off prior to final analysis commencing. Thematic analysis of qualitative data will be carried out using a constant comparison method of analysis, which will gather and generate ideas and categories through inductive processes. The computer package NVivo will be used to support the primary data analysis [49]. Memo writing will be carried out to describe details of the interview setting and interaction of respondent and interviewer that may not be captured in audio transcriptions. This thematic analysis has deductive and inductive elements, lending itself to multidisciplinary health research [50]. The analysis framework will incorporate the key theoretical constructs and respond to the context of policy and practice to include a range of deductive themes. Further themes will be induced from the interview data.

An appropriate balance of integration between empirical data and interpretation will be ensured. The investigators will extract the meaning of the empirical data and interpret them whilst acknowledging the complexity of the phenomena of CVD risk reduction in the context of community engagement [51]. This method holds links to the original data and the output allows comprehensive and transparent data analysis.

\section{Discussion}

Given that the rolling out of the NHS Health Checks programme over and above current care across the UK has not been implemented as efficiently as it could have been, especially in some of the most disadvantaged groups prone to developing CVD, the project aims to scale-up packages of interventions for cardiovascular prevention particularly to these vulnerable populations. This interdisciplinary project includes public health, social and behavioural science approaches. The main focus of this project is the de-institutionalization of health care by operating outside of formal healthcare settings. The project will place emphasis on the power of citizens, combining their efforts to generate cultures of care which complement or even compensate for the inadequacies of formal systems and are thus more sustainable. The research project will ultimately develop a community engagement-based CVD primary prevention programme to support or enhance the performance of the NHS health care.

We have considered the potential operational issues in performing the study and have carefully thought out mechanisms to mitigate these. The following potential practical

\section{Context}

Contextual factors that shape theories of how the intervention works

Contextual factors that affect (and may be affected by) implementation, intervention mechanisms and outcomes

Causal mechanisms present within the context which act to sustain the status quo, or potentiate effects

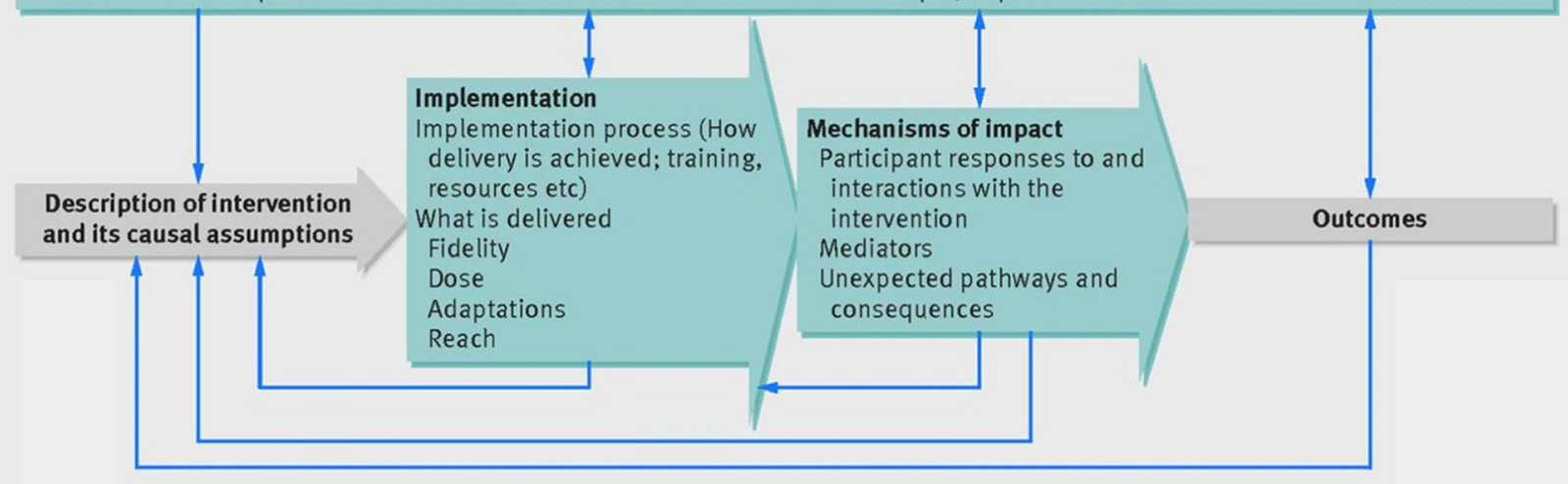

Fig. 1 Process evaluation model by Moore et al. [45] 
challenges in implementing the project have been identified: retaining the volunteers, retaining the research participants, and maintaining the quality of goal setting and motivational interviewing for an effective behaviour change intervention. In order to retain the volunteers, we will recruit staff on a paid basis from each site to work closely with the volunteers. We will conduct risk assessment audits together with the site staff so that additional needs of the volunteers, generated by their participation in the study, can be met. The research team will liaise with the paid site staff to take steps to ensure the volunteers are engaged and enthusiastic about their role and support them when this is not the case. In order to ensure retention of the research participants, the researchers will work very closely with the volunteers. The researcher will be present in a number of motivational interviewing sessions (with participants' consent) and become familiar with the participants' experiences and circumstances. As the participants come from a low socio-economic background it is likely that they might drop out from the study due to other pressures in their everyday lives. We will try to identify such potential threats among the participants and encourage the volunteers to pre-empt any lack of engagement by discussing strategies for maintaining motivation with their participants. The motivational interviews will be quality checked at intervals by researchers and trainers by observing some of these sessions (with participants' consent). A regular feedback mechanism and tailored training plan are in place to improve the interview quality when any weakness is identified. With this carefully designed risk mitigation plan we hope to make sure the project meets its goals.

With our approach of taking cardiovascular disease prevention out of formal healthcare services and bringing it into the control of people in their own communities we hope to co-design a sustainable and scalable intervention which enables members of low SES communities in the UK to take control of their cardiovascular health, and improve their quality of life.

\section{Acknowledgements}

We would like to thank European Commission, Horizon 2020 Research and Innovation Action Grant for providing us with the fund to execute this research project.

\section{Trial registration number}

ISRCTN68334579 (https://doi.org/10.1186/ISRCTN68334579).

\section{Authors' contributions}

PN has designed the protocol and written the manuscript. HvM, LG, GM, SA, $E F, S B, M B, J Y L R, T S$, and $H B$ provided inputs in the study design. All authors read and approved the final contextual protocol manuscript.

\section{Funding}

This protocol is a contextual plan for the SPICES project in the UK. The SPICES project received funding from the European Commission through the Horizon 2020 Research and Innovation Action Grant Agreement No 733356 to implement and evaluate a comprehensive CVD prevention programme in five settings: a rural \& semi-urban community in a low-income country (Uganda), middle income (South Africa) and vulnerable groups in three high- income countries (Belgium, France and United Kingdom). The funder had no role in the design, decision to publish, or preparation of the manuscript.

\section{Availability of data and materials}

Not applicable because a protocol should not contain any data; it sets out the research questions and how they will be addressed.

\section{Ethics approval and consent to participate}

This protocol has received ethics approval from the University of Sussex, The BSMS Research Governance and Ethics Committee (RGEC (ER/BSMS9E3G/1)), and from Nottingham Trent University (CREC2018/286). All participants will be requested to consent before enrolment into the study. All participant information will be kept confidential and accessible only to the key investigative team. All published data will be anonymised and can be accessed based on a written request to the Principal Investigator.

\section{Consent for publication}

Not Applicable because this manuscript does not content any individual person's data in any form.

\section{Competing interests}

The authors declare that they have no competing interests.

\section{Author details}

'Department of Global Health and Infection, Brighton and Sussex Medical School, The University of Sussex, Brighton, UK. ${ }^{2}$ Department of Primary Care and Public Health, Brighton and Sussex Medical School, The University of Sussex, Brighton, UK. ${ }^{3}$ School of Social Sciences, Nottingham Trent University, Nottingham, UK. ${ }^{4}$ Department of Disease Control and Environmental Health, School of Public Health, College of Health Sciences, Makerere University, Kampala, Uganda. ${ }^{5}$ Department of Primary and Interdisciplinary Care, University of Antwerp, Antwerp, Belgium. ${ }^{6}$ EA 7479 SPURBO, Faculté de Médecine et des Sciences de la Santé, Université de Bretagne Occidentale, Brest, France. 'Department of Psychology, University of Limpopo, Mankweng, South Africa.

Received: 4 August 2019 Accepted: 5 January 2020

Published online: 12 March 2020

\section{References}

1. World Health Organisation; WHO. Global Health Observatory (GHO) data. 2017. Retrieved from https://www.who.int/gho/mortality_burden_disease/ causes_death/top_10/en. Accesses 18 May 2019.

2. British Heart Foundation report BHF. https://www.bhf.org.uk informationsupport/publications/policy-documents/annual-report-2017. Accessed 20 Oct 2019

3. Amuzu A, Carson C, Watt HC, Lawlor DA, Ebrahim S. Influence of area and individual life course deprivation on health behaviours: findings from the British Women's heart and Health study. Eur J Cardiovasc Prev Rehabil. 2009; 16(2):169-73. https://doi.org/10.1097/HJR.0b013e328325d64d.

4. Public Health England (2013). NHS Health Check implementation review and action plan Summary, https://assets.publishing.service.gov.uk/government/ uploads/system/uploads/attachment_data/file/224536/NHS_Health_Check_ implementation_review_and_action_plan_summary_web.pdf. Accessed 1 May 2019

5. QRISK-2, 2017, cardiovascular disease risk calculator. Retrieved from https:// grisk.org/2017/. Accessed 1 May 2019.

6. Public Health, England. NHS Health Checks 'prevented thousands of heart attacks' 2016. Retrieved from https:/www.nhs.uk/news/heart-and-lungs/nhshealth-checks-prevented-thousands-of-heart-attacks/. Accessed 1 May 2019.

7. Public Health England. Factsheet: Implementation of the NHS Health Check programme. 2014. https://www.england.nhs.uk/wp-content/uploads/2014/ 02/pm-fs-3-1.pdf. Accessed 1 May 2019.

8. Waterall Jamie. PHE. Getting Serious About CVD Prevention. Reducing Variation \& Optimising Care. 2018. https://www.healthcheck.nhs.uk/ seecmsfile/?id=195. Accessed 1 May 2019.

9. Dalton AR, Bottle A, Okoro C, Majeed A, Millett C. Uptake of the NHS Health checks programme in a deprived, culturally diverse setting: cross-sectional study. J Public Health (Oxf). 2011;33(3):422-9. https://doi.org/10.1093/pubmed/fdr034. 
10. Robson J, Dostal I, Sheikh A, et al. The NHS Health Check in England: an evaluation of the first 4 years. BMJ Open. 2015;6:e008840. https://doi.org/10. 1136/bmjopen-2015-008840.

11. Emma H, MacLure C, Martin A, Saunders CL, Meads C, Walter FM, Griffin SJ, Mant J, Usher-Smith JA. Reasons why people do not attend NHS Health checks: a systematic review and qualitative synthesis. BJGP. 2018;68(666): e28-35. https://doi.org/10.3399/bjgp17X693929.

12. CTSA. Principles of Community Engagement, Second Edition, NIH Publication No. 11-7782. 2011. https://atsdr.cdc.gov/ communityengagement/pdf/PCE_Report_508_FINAL.pdf. Accessed 1 May 2019

13. O'Mara-Eves A, Brunton G, McDaid D, Oliver S, Kavanagh J, Jamal F, Matosevic T, Harden A, Thomas J. Community engagement to reduce inequalities in health: a systematic review, meta-analysis and economic analysis. Public Health Res. 2013;1:4

14. Bird JA, McPhee SJ, Ha NT, Le B, Davis T, Jenkins CN. Opening pathways to cancer screening for Vietnamese-American women: lay health workers hold a key. Prev Med. 1998;27(6):821-9.

15. Gaziano Thomas A, Abrahams-Gessel S k, Denman CA, Montano CM, Khanam M, Puoane T, Levitt NS. An assessment of community health workers' ability to screen for cardiovascular disease risk with a simple, non invasive risk assessment instrument in Bangladesh, Guatemala, Mexico, and South Africa: an observational study. Lancet Glob Health. 2015;3:e556-63. https://doi.org/10.1016/S2214-109X(15)00142-4.

16. Wood J. The University as a public good: active citizenship and university community engagement. Int J Progress Educ. 2012;8(3):15-31 Retrieved from http://dergipark.org.tr/ijpe/issue/26312/277299.

17. Olivr,D., Foot, C., Hamphries, R. Kings Fund:Making.Out Helath and Care Systems fit for an aging population 2011. Retrieved from, https://www. kingsfund.org.uk/sites/default/files/Noluntary-and-community-sector-inhealth-implications-NHS-reforms-The-Kings-Fund-june-2011_0.pdf. Accessed 1 May 2019

18. Abel J, Bowra J, Walter T, et al. Compassionate community networks: supporting home dying. BMJ Support Palliat Care. 2011;1:129-33. https:// doi.org/10.1136/bmjspcare-2011-000068.

19. Baum FE, Ziersch AM. Social capital. J Epidemiol Community Health. 2003:57:320-3.

20. Libby SL, Richardson H, Murray S, Kellehear A. Understanding the impact of a new public health approach to end of life care: a qualitative study of a community-led intervention. Lancet. 2017;389(Special issue):788. https://doi. org/10.1016/S0140-6736(17)30484-1.

21. Boyd, McKernon S, Mullin B, Old A. Improving healthcare through the use of co-design. N Z Med J. 2012;125:1357

22. Geofrey M, Wanyenze RK, Ndejjo R, Ssinabulya I, van Marwijk H, Ddumba I, Bastiaens H, Nuwaha F. An implementation science study to enhance cardiovascular disease prevention in Mukono and Buikwe districts in Uganda: a stepped-wedge design. Health Serv Res. 2019;19:253. https://doi. org/10.1186/s12913-019-4095-0.

23. Glasgow RE, McKay HG, Piette JD, Reynolds KD. The RE-AIM framework for evaluating interventions: what can it tell us about approaches to chronic illness management? Patient Educ Couns. 2001;44:119-27.

24. Theobald S, Brandes N, Gyapong M, et al. Implementation research: new imperatives and opportunities in global health. Lancet. 2018;392:2214-28.

25. Peters $\mathrm{DH}$, Adam $\mathrm{T}$, Alonge $\mathrm{O}$, et al. Implementation research: what it is and how to do it. BMJ. 2013;347:f6753.

26. Brown $\mathrm{CH}$, Curran $\mathrm{G}$, Palinkas LA, et al. An overview of research and evaluation designs for dissemination and implementation. Annu Rev Public Health. 2017;38:1-22

27. Greene JC. Mixed Methods in Social Inquiry. San Francisco: Jossey-Bass; 2007.

28. Five-Step Approach to Stakeholder Engagement (BSR Report). (2011). Retrieved from www.bsr.org/reports/BSR_Stakeholder_Engagement_ Stakeholder_Mapping.final.pdf. Accessed 1 May 2019.

29. NICE Public health guideline [PH6] 'behaviour change: general approaches' 2007 Retrieved from https://www.nice.org.uk/guidance/ph6/resources/ behaviour-change-general-approaches-pdf-55457515717. Accessed 1 May 2019

30. Damschroder Laura J, Aron DC, Keith RE, Kirsh SR, Alexander JA, Lowery JC. Fostering implementation of health services research findings into practice: a Consolidated Framework for Advancing Implementation Science. Implement Sci. 2009;4:50. https://doi.org/10.1186/1748-5908-4-50.

31. Susan $M$, Richardson $M$, Johnston $M$, et al. The behaviour change technique taxonomy ( $\mathrm{v} 1$ ) of 93 hierarchically clustered techniques: building an international consensus for the reporting of behavior change interventions. Ann Behav Med. 2013:46(1):81-95. https://doi.org/10.1007/s12160-013-9486-6.

32. Susan M, Van Stralen MM, West R. The Behaviour Change Wheel: A New Method for Characterising and Designing Behaviour Change Interventions. Implement Sci. 2011;6(1):42.

33. Salim Y, Hawken S, Ônpuu S, on behalf of the INTERHEART Study Investigators, et al. Effect of potentially modifiable risk factors associated with myocardial infarction in 52 countries (the INTERHEART study): a casecontrol study. Lancet. 2004;364:937-52.

34. Sacks FM, Svetkey LP, Vollmer WM, et al. Effects on blood pressure of reduced dietary sodium and the dietary approaches to stop hypertension (DASH) diet. N Engl J Med. 2001;344:3-10. https://doi.org/10.1056/NEJM200101043440101.

35. Woringer $M$, Nielsen JJ, Zibarras $L_{\text {, }}$ et al. Development of a questionnaire to evaluate patients' awareness of cardiovascular disease risk in England's National Health Service Health Check preventive cardiovascular programme. BMJ Open. 2017;7:e014413. https://doi.org/10.1136/bmjopen-2016-014413.

36. Aleksandra L, Scholz U, Schwarzer R. The General Self-Efficacy Scale: Multicultural Validation Studies. J Psychol. 2005;139(5):439-57. https://doi. org/10.3200/JRLP.139.5.439-457.

37. Kontopantelis E, Mamas MA, Van Marwijk H, Ryan AM, Buchan IE, Ashcroft DM, et al. Geographical epidemiology of health and overall deprivation in England, its changes and persistence from 2004 to 2015: A longitudinal spatial population study. J Epidemiol Community Health. 2018;72(2):140-7.

38. Hooper R, Teerenstra S, de Hoop E, Eldridge S. Sample size calculation for the stepped wedge and other longitudinal cluster randomised trials. Stat Med. 2016:35:4718-28.

39. Salim Y, Rangarajan S, Teo K, Islam S, et al. Cardiovascular. Risk and events in 17 low-, middle-, and high-income countries. N Engl J Med. 2014;371:81827. https://doi.org/10.1056/NEJMoa1311890.

40. Joseph WN, Lloyd A, Edwards A, Stobbart L, Tomson D, Macphail S, Dodd C, Brain K, Elwyn G, Thomson R. Implementing shared decision making in the NHS: lessons from the MAGIC programme. BMJ. 2017;357:j1744. https://doi. org/10.1136/bmj.j1744.

41. Guest G, Bunce A, Johnson L. How many interviews are enough?: an experiment with data saturation and variability. Field Methods. 2006;18(1): 59-82. https://doi.org/10.1177/1525822X05279903.

42. Malterud K, Siersma VD, Guassora AD. Sample size in qualitative interview studies: guided by information power. Qual Health Res. 2016:26(13):1753-60. https://doi.org/10.1177/1049732315617444.

43. Bellg AJ, Borrelli B, Resnick B, Hecht J, Minicucci DS, Ory M, Treatment Fidelity Workgroup of the NIH Behavior Change Consortium, et al. Enhancing Treatment Fidelity in Health Behavior Change Studies: Best Practices and Recommendations From the NIH Behavior Change Consortium. Health Psychol. 2004:23(5):443-51. https://doi.org/10.1037/02786133.23.5.443.

44. Thirsk LM, Clark AM. Using Qualitative Research for Complex Interventions: The Contributions of Hermeneutics. Int J Qual Methods. 2017:16:1-10. https://doi.org/10.1177/1609406917721068.

45. Graham M, Audrey S, Barker M, Bond L, Bonell C, Hardeman W, Moore L, O'Cathain A, Tinati T, Wight D, Baird J. Process evaluation of complex interventions: Medical Research Council guidance. BMJ. 2015;350:h1258. https://doi.org/10.1136/bmj.h1258.

46. Campbell MK, Piaggio G, Elbourne DR, Altman DG, for the CONSORT Group Consort 2010 statement: extension to cluster randomised trials. BMJ. 2012; 345:e5661 PMID: 22951546.

47. StataCorp. Stata statistical software: release 16. College Station: StataCorp LLC; 2019.

48. StataCorp. Stata statistical software: release 15. College Station: StataCorp LLC; 2017

49. Jane R, Lewis J, Nicholls CMN, Ormston R. Qualitative research practice: a guide for social science students and researcher. London: Sage Publications; 2013.

50. Gale NK, Heath G, Cameron E, Rashid S, Redwood S. Using the framework method for the analysis of qualitative data in multi-disciplinary health research. BMC Med Res Methodol. 2013;13:117. https://doi.org/10.1186/1471-2288-13-117.

51. Green J, Thorogood N. Qualitative methods for health research. London: Sage Publications Ltd.; 2005. 